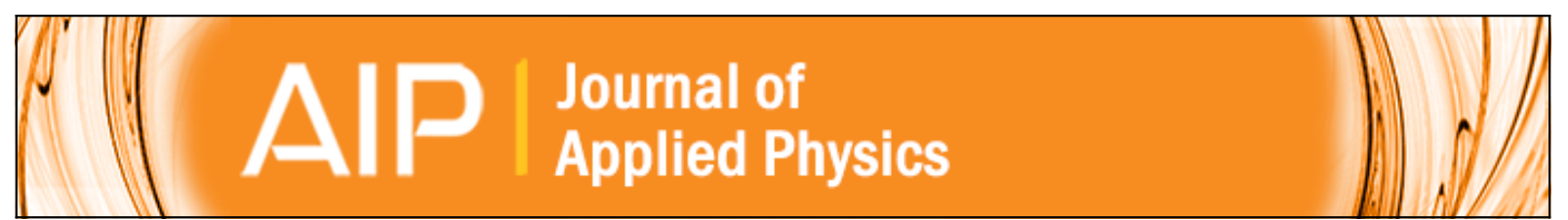

Quasi-static magnetic measurements to predict specific absorption rates in magnetic fluid hyperthermia experiments

D. F. Coral, P. Mendoza Zélis, M. E. de Sousa, D. Muraca, V. Lassalle, P. Nicolás, M. L. Ferreira, and M. B. Fernández van Raap

Citation: Journal of Applied Physics 115, 043907 (2014); doi: 10.1063/1.4862647

View online: http://dx.doi.org/10.1063/1.4862647

View Table of Contents: http://scitation.aip.org/content/aip/journal/jap/115/4?ver=pdfcov

Published by the AIP Publishing

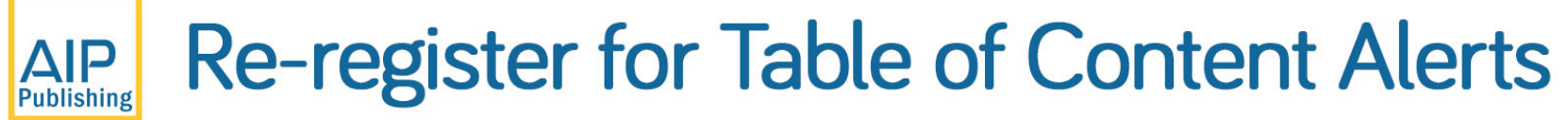




\title{
Quasi-static magnetic measurements to predict specific absorption rates in magnetic fluid hyperthermia experiments
}

\author{
D. F. Coral, ${ }^{1}$ P. Mendoza Zélis, ${ }^{1}$ M. E. de Sousa, ${ }^{1}$ D. Muraca, ${ }^{2}$ V. Lassalle, ${ }^{3}$ P. Nicolás, ${ }^{3}$ \\ M. L. Ferreira, ${ }^{3}$ and M. B. Fernández van Raap ${ }^{1, a)}$ \\ ${ }^{1}$ Instituto de Física La Plata (IFLP-CONICET), Departamento de Física, Facultad de Ciencias Exactas, \\ Universidad Nacional de La Plata (UNLP), c.c. 67, 1900 La Plata, Argentina \\ ${ }^{2}$ Instituto de Física "Gleb Wataghin" (IFGW), Universidade Estadual de Campinas, Brazil \\ ${ }^{3}$ Instituto de Química del Sur (INQUISUR, UNS-CONICET), Avda. Alem 1253, B8000CPB, Bahía Blanca, \\ Argentina
}

(Received 11 October 2013; accepted 7 January 2014; published online 23 January 2014)

\begin{abstract}
In this work, the issue on whether dynamic magnetic properties of polydispersed magnetic colloids modeled using physical magnitudes derived from quasi-static magnetic measurement can be extrapolated to analyze specific absorption rate data acquired at high amplitudes and frequencies of excitation fields is addressed. To this end, we have analyzed two colloids of magnetite nanoparticles coated with oleic acid and chitosan in water displaying, under a radiofrequency field, high and low specific heat power release. Both colloids are alike in terms of liquid carrier, surfactant and magnetic phase composition but differ on the nanoparticle structuring. The colloid displaying low specific dissipation consists of spaced magnetic nanoparticles of mean size around $4.8 \mathrm{~nm}$ inside a large chitosan particle of $52.5 \mathrm{~nm}$. The one displaying high specific dissipation consists of clusters of magnetic nanoparticles of mean size around $9.7 \mathrm{~nm}$ inside a chitosan particle of $48.6 \mathrm{~nm}$. The experimental evaluation of Néel and Brown relaxation times $\left(\sim 10^{-10} \mathrm{~s}\right.$ and $10^{-4} \mathrm{~s}$, respectively) indicate that the nanoparticles in both colloids magnetically relax by Néel mechanism. The isothermal magnetization curves analysis for this mechanism show that the magnetic nanoparticles behave in the interacting superparamagnetic regime. The specific absorption rates were determined calorimetrically at $260 \mathrm{kHz}$ and up to $52 \mathrm{kA} / \mathrm{m}$ and were well modeled within linear response theory using the anisotropy density energy retrieved from quasi-static magnetic measurement, validating their use to predict heating ability of a given polydispersed particle suspension. Our findings provide new insight in the validity of quasi-static magnetic characterization to analyze the high frequency behavior of polydispersed colloids within the framework of the linear response and Wohlfarth theories and indicate that dipolar interactions play a key role being their strength larger for the colloid displaying higher dissipation, i.e., improving the heating efficiency of the nanoparticles for magnetic fluid hyperthermia. (C) 2014 AIP Publishing LLC. [http://dx.doi.org/10.1063/1.4862647]
\end{abstract}

\section{INTRODUCTION}

Magnetic hyperthermia is nowadays being settled as a therapeutic for cancer treatment. Abnormally high temperature is induced in a tumor using magnetic nanoparticles (MNPs) and radiofrequency (RF) field to selectively kill tumor cells. After the first pioneer work on Limph Node, ${ }^{1}$ latter resumed by Jordan's group, ${ }^{2}$ much effort has been devoted to enhance nanoparticles properties preserving biological requirements (i.e., non-toxicity and stability in suspension in biological medium). MNPs displaying large specific heating, usually characterized by the specific absorption rate (SAR), are desired to lower dose for treatment. ${ }^{3}$ Although large number of particles and coatings has been studied, iron oxides (magnetite and maghemite) coated with organic molecules dispersed in water are widely accepted as the colloid displaying the best biocompatibility. Most common used surfactants include non saturated monocarboxylic

\footnotetext{
a) Author to whom correspondence should be addressed. Electronic mail: raap@fisica.unlp.edu.ar. Phone: +54 2214246062 x 257. Fax: +54 221 4236335 .
}

acids, ${ }^{4}$ such as oleic, lauric, myristic and stearic acids, among others. ${ }^{5}$ Polymeric coating also appears as a suitable alternative, especially Chitosan which is a natural biodegradable polymer with a high density of amine groups in their structure that may be protonated depending on $\mathrm{pH}$ to achieve stability in suspension. Colloids formed by MNPs coated with polyethylene glycol, ${ }^{6}$ polyethylene glycol fumarate, polyvinyl alcohol, acrylate-based coatings, dextran-based coatings, synthetic polyesters, ${ }^{7}$ alginate and polyethylenimine, have been reported. ${ }^{8}$ Besides the achieved stability, other main difference between monocarboxylic acids and polymeric coatings, of importance for magnetic hyperthermia application, resides on coated nanoparticles hydrodynamic size. In the former case, a small molecule is bound to the MNP surface then the overall nanoparticle size increase is small. In the latter a large macromolecule is bonded resulting in a large overall nanoparticle size and consequently in a large hydrodynamic size.

Although much knowledge and many assays have been used to synthesize, test and characterize various MNPs type for magnetic hyperthermia, many questions remain still open. First, it is unclear if rather larger dissipation would be 
obtained by Néel mechanism using particles of large hydrodynamic size or by Brown mechanism using particles of smaller sizes. Second, the influence of interparticle dipolar interactions on SAR is not clearly understood either. Experimental results indicating that increasing dipolar interactions increase and decrease the SAR have been published. Increases in dipolar interaction strength were attempted in 30-33 nm $\mathrm{MFe}_{2} \mathrm{O}_{4}(\mathrm{M}=\mathrm{Mg}, \mathrm{Ni}, \mathrm{Co})$ spinel ferrite nanoparticles by increasing the size distribution and it was shown that SAR, and minor loops area increase with increasing distribution standard deviation (s.d). ${ }^{9}$ However, increasing s.d effects arising from size dependence cannot be discriminated from dipolar effects. On the other hand, experimental studies on monodispersed maghemite nanoparticles of $12 \mathrm{~nm}$ magnetic size and $14 \mathrm{~nm}$ hydrodynamic size evidence that an increase in concentration leads to a decrease in MNP's heating capacity ${ }^{10}$ and this result was later supported by Monte Carlo simulations. ${ }^{11}$ More recently, high frequency hysteresis loops measurements of various monodispersed $\mathrm{Fe}_{\mathrm{x}} \mathrm{C}_{\mathrm{y}}$ (core-shell) and $\mathrm{FeCo}$ samples displaying same size but different anisotropy has been reported. ${ }^{12}$ The authors showed that an increase in dipolar interaction strength (achieved by increasing anisotropy) improve heating efficiency in a colloidal solution when nanoparticles are free to arrange in chains and columns. We have recently showed for aqueous stable suspensions of magnetite MNPs coated with a monocarboxyl acid (citric acid), that interparticle interactions are relevant influencing the magnitude of Neél relaxation time and then dissipation mechanism selection. ${ }^{13}$

For single-domain MNP, coherent magnetization reversal is due to Néel and/or Brown processes. The magnetization lags behind the field when the nanoparticle magnetic moment switches (Neél) or when the particle itself rotates in the medium (Brown). Theoretical treatments ${ }^{14,15}$ of these mechanisms for magnetic hyperthermia application are based on linear response ${ }^{16}$ and Stoner Wohlfarth theories. ${ }^{17}$ Briefly, when a randomly oriented assembly of uniaxial single-domain particles of volume $V$ and specific saturation magnetization $\mathrm{M}_{\mathrm{s}}$ is subjected to a RF field of frequency $f$ and amplitude $H_{0}$, SAR is given by

$$
S A R=\mu_{0} \pi f H_{o}^{2} \chi^{\prime \prime}(f, \tau),
$$

where $\mu_{0}$ is the permeability of free space $\left(4 \pi \times 10^{-7} \mathrm{H} / \mathrm{m}\right)$ and $\chi^{\prime \prime}(f)$ is the out-of-phase susceptibility given by

$$
\chi^{\prime \prime}(f)=\frac{2 \pi f \tau}{1+(2 \pi f \tau)^{2}} \chi_{0}
$$

and $\chi_{0} \cong \frac{\mu_{0} \rho M_{s}^{2} V}{3 k_{B} T}$ is the d.c. mass susceptibility. The dynamic of the particle's dipole moment is governed by the effective relaxation time $\tau$ given by $\tau^{-1}=\tau_{N}^{-1}+\tau_{B}^{-1}$ when independency between modes is assumed. The relaxation times depend on medium viscosity $(\eta)$ and hydrodynamic volume $V_{H}$ through Brown component given by $\tau_{B}=\frac{3 \eta V_{H}}{k_{B} T}$, and on magnetic volume, effective anisotropy energy density $K_{e}$ and attempt time $\tau_{0} \sim 10^{-10} \mathrm{~s}$ (Ref. 18) through Néel given by $\tau_{N}=\tau_{0} \exp \left(E_{a} / k_{B} T\right)$. The energy barrier $E_{a}$ of $\mathrm{a}$ double-well potential is related to $K_{e} V$. Equation (1) holds for single-size isolated particles and its applicability has been tested for nearly monodispersed nanoparticles, relative s.d. lower than 0.18 , produced by thermal decomposition. ${ }^{19}$ However, the more complex case of a polydisperse assembly of nanoparticles, commonly obtained from coprecipitation methods, has not been fully studied. Recently, we show for cubic-like shaped $\mathrm{Zn}_{\mathrm{x}} \mathrm{Fe}_{3-\mathrm{x}} \mathrm{O}_{4}$ particles $(0.0 \leq \mathrm{x} \leq 0.5)$ that normalized SAR data of a large set of colloids merge into a single curve consistent with the frequency factor $\chi^{\prime \prime}(f) / \chi_{0}$, but the SAR experimental values were not theoretically reproduced. ${ }^{20}$ The importance of MNPs size distribution on SAR has been pointed out theoretically ${ }^{14,21}$ and experimentally. ${ }^{22,23}$ Theoretical calculation has indicated that the useful $H_{0}$ and $f$ values strongly depend on size distribution parameters ${ }^{22}$ and predicted a reduction close to $70 \%$ in the SAR when size polydispersity increase from monodispersed (lognorm deviation $\sigma=0$ ) to $\sigma=0.4$ at optimum mean size. ${ }^{14,21}$ Salas et al. ${ }^{23}$ have measured at $18 \mathrm{~nm}$ magnetite coated with DMSA a SAR value three times lower when the size distribution s. d. was duplicated.

Here, aqueous colloids displaying a wide magnetic-core size distribution are used to test the applicability and validity of Eq. (1) to predict heating ability of polydispersed suspensions of particle when their dynamics are probed by isothermal magnetization, i.e., using quasi static measurements. The dispersed phase is magnetite coated with oleic acid and chitosan. We first used several structural techniques like FTIR, TEM, AFM, SAXS, and DLS to completely characterize the nanoparticles and their aqueous suspensions, and DC magnetometry to determine magnetic properties. Once the structural and magnetic parameters are known, we analyze SAR theoretical behavior.

Articles regarding magnetite dispersion containing chitosan synthesis protocols and their physicochemical and magnetic characterization have been reported. ${ }^{24,25}$ However, biomedical applications of these materials were not evaluated. One article deals with the preparation of stable colloidal magnetic dispersions composed of magnetite and chitosan by two steps crosslinking of polymeric networks ${ }^{24}$ resulting in $\mathrm{Fe}_{3} \mathrm{O}_{4}$-chitosan magnetic composite nanoparticles with a core-shell structure and a diameter of $30-50 \mathrm{~nm}$. The inductive heating for hyperthermia applications was accomplished but no quantitative evaluation of SAR or any analysis to relate heating efficiency to magnetic and structural properties were attempted.

\section{MATERIALS AND METHODS}

\section{A. Synthesis of stable aqueous suspension of $\mathrm{Fe}_{3} \mathrm{O}_{4}$ coated with oleic acid and chitosan}

All chemicals used in this work are of analytical grade. Oleic acid (OA) was from Anedra, Chitosan (CS) from Chitoclear, by Primex (Iceland) and solvents from Dorwill (Argentina, SA).

The colloids were prepared by co-precipitation method ${ }^{26}$ commonly used to prepare magnetite, with some modifications. ${ }^{10}$ The same amount of CS $(450 \mathrm{mg})$ was used to hydrophilize $300 \mathrm{mg}$ of oleic-acid coated magnetite core (OA-core) 
nanoparticles $(3 / 2$ of CS/OA-core $w / w)$ previously synthesized with different mass ratio of oleic acid to magnetite (1/1 and 4/1), In brief, $\mathrm{FeCl}_{3} \cdot 6 \mathrm{H}_{2} \mathrm{O}\left(0.0121 \mathrm{~mol}\right.$ of $\left.\mathrm{Fe}^{3+}\right)$ and $\mathrm{FeSO}_{4}\left(6.46 \times 10^{-3} \mathrm{~mol} \mathrm{Fe}{ }^{2+}\right)$ were dissolved in $100 \mathrm{ml}$ of distilled water. The corresponding proportion of OA was incorporated to the mixture. Then, $25 \mathrm{ml}$ of $\mathrm{NaOH} 5 \mathrm{M}$ were added to precipitate the oxide. This addition was performed at controlled rate to minimize the magnetite aggregation. The mixture was allowed to complete the magnetite formation during $30 \mathrm{~min}$. The obtained dark solution was decanted and supernatant was extracted. Then, bidistilled water was added to remove the impurities by filtration. These washing cycles were repeated three times to ensure complete elimination of the ionic moieties. The solid was dried in an oven at $45^{\circ} \mathrm{C}$ overnight under vacuum. In a second step, the nanoprecipitation method was adopted to coat OA-core with $\mathrm{CS}^{27}$ In brief about $300 \mathrm{mg}$ of OA-core were dispersed in acetone under ultrasound treatment during $15 \mathrm{~min}$. Then, the corresponding volume of a solution of $9.73 \mathrm{mg} / \mathrm{ml}$ of CS in acetic acid (50\%) was incorporated. The solid MNPs decanted immediately. The supernatant was removed and the solid re-dispersed in distilled water. At these conditions, a dispersion stable during almost three months was formed. A deeper discussion on synthesis protocol and physicochemical characterization can be found elsewhere. ${ }^{28}$ From these procedures carried out varying synthesis parameters, we choose two colloids displaying high and low SAR, here in after labeled as $\mathrm{HD}$ and $\mathrm{LD}$, respectively.

\section{B. Structural and physical-chemical properties}

Fourier Transform Infra Red (FTIR) Spectroscopy was used to ensure polymeric coating accomplishment. A FTIR (DRIFTS) Thermo Scientific Nicolet 6700 spectrometer was used for recording spectra in the range $4000-400 \mathrm{~cm}^{-1}$. A few milligrams of the samples (roughly $10-20 \mathrm{mg}$ ) were mixed in a mortar manually with near $50 \mathrm{mg}$ of $\mathrm{KBr}$ powder. The mixture was placed in the sample unit of FTIR-DRIFTS.

For AFM analysis the colloids were diluted to $1 / 10000$ from their original concentration $[\mathrm{x}]$ to ensure single CS particle observation and were dropped on newly peeled muscovite mica sheet V-1 grade (SPI Supplies) and dried in an oven at $40{ }^{\circ} \mathrm{C}$. The samples were imaged in air and at room temperature using standard semicontact mode in a NT-MDT Solver Pro microscope equipped with a APPNANO-ACTA silicon probe consistent for high resolution imaging. The rectangular cantilever of $40 \mathrm{~N} / \mathrm{m}$ force constant and $281.8 \mathrm{kHz}$ resonant frequency holds a sharp tip of $6 \mathrm{~nm}$ curvature radius.
Transmission electron microscopy measurements (TEM) were carried out with a $300 \mathrm{keV}$ JEM 3010 microscope to obtain particle size distribution by drying the aqueous dispersions on carbon coated copper grids.

XRD was carried out for phase identification. The patterns were recorded at room temperature using a PHILIPS PW1710 diffraction spectrometer. Small-angle X-ray scattering (SAXS) was performed to determine nanoparticle structuring in suspension using synchrotron radiation. The data were obtained at a wavelength of $\lambda=1.822 \AA$ $(\varepsilon=6.804 \mathrm{keV})$, using MAR $165 \mathrm{CCD}$-detector and corrected for absorption, background due to solvent density fluctuation scattering prior to the fitting procedure. The momentum transfer vector $q=4 \pi \sin (\theta) / \lambda$, being $2 \theta$ the scattering angle, range from 0.06 to $2.0 \mathrm{~nm}^{-1}$ was achieved by measuring with two sample-detector distances of 975 and $1977 \mathrm{~mm}$. Absolute scale to express scattered intensity as differential cross section $\left(\mathrm{cm}^{-1}\right)$ was determined using water as a secondary standard. For measurement the as prepared colloids were sonicated and injected in between two clear Ruby Mica Discs, $25 \mu \mathrm{m}$ thick each, of the liquid sample holder.

Nanoparticles hydrodynamic diameter $\left(D_{H}\right)$ was retrieved from Dynamical Light Scattering (DLS). Colloidal system stability is analyzed from Z-potential $(\zeta)$ obtained from Laser Doppler Electrophoresis measurements. A Malvern Zetasizer nano ZS90 device was employed. Dispersions of magnetic MNPs in bidistilled water were prepared in a proportion of $0.1 \mathrm{mg} \mathrm{MNPs} / \mathrm{mL}$ at $\mathrm{pH}=5.5$ to measure $\zeta$. The same dispersions were ultrasonicated during $1 \mathrm{~h}$ to measure the average particle sizes.

Atomic Emission Spectroscopy with Induced Plasma Coupled, (ICP-AES), simultaneous high resolution Shimadzu 9000 was utilized to determine the MNP's composition in terms of total $\mathrm{Fe}$ content. Alternatively, a common and standardized chemical volumetric analysis, with $\mathrm{K}_{2} \mathrm{Cr}_{2} \mathrm{O}_{7}$ used as titrant, was used for colloid concentration $[\mathrm{x}]$ determination, expressed as magnetite mass per solvent volume with an accuracy of $2 \%$. [x] values were used to calculate SAR values from calorimetric data and to normalize magnetic measurements dividing by magnetite mass concentration $[\mathrm{m}]=[\mathrm{x}] \mathrm{V} / \mathrm{M}_{\mathrm{p}}$. The nanoparticle mass $\left(\mathrm{M}_{\mathrm{p}}\right)$ was obtained drying $\mathrm{V}=100 \mu \mathrm{l}$ of colloid at $37^{\circ} \mathrm{C}$ during $24 \mathrm{~h}$. Values of [x] and [m] are reported in Table I.

\section{Magnetic measurements}

MNP's magnetic response was measured by DC. Magnetometry, understood as quasi-static magnetic measurements comparing with the condition of magnetic hyperthermia

TABLE I. Nanoparticles and colloids composition from nominal precursors proportions: OA/core is the mass radio of oleic acid to magnetite core and $\mathrm{CS} /$ core-OA the mass radio of chitosan to OA coated magnetite nanoparticle $(\mathrm{OA} / \mathrm{core})$ and from $\mathrm{ICP}$ and titration: $[\mathrm{x}]$ is the concentration expressed as $\mathrm{Fe}_{3} \mathrm{O}_{4}$ mass per $\mathrm{ml}$ of water and $[\mathrm{m}]$ the mass of $\mathrm{Fe}_{3} \mathrm{O}_{4}$ over nanoparticle mass. Magnetic colloids zeta potential ( $\zeta$ ) before chitosan coating (i.e., OA/core) and of final colloid after CS coating.

\begin{tabular}{lcccccc}
\hline \hline Label & $\mathrm{OA} / \mathrm{core}$ & $\mathrm{CS} / \mathrm{core}-\mathrm{OA}$ & {$[\mathrm{x}] \mathrm{mg} / \mathrm{mL}$} & {$[\mathrm{m}] \mathrm{mg}_{\mathrm{Fe} 3 \mathrm{O} 4 / \mathrm{mg}_{\mathrm{NP}}}$} & $\zeta_{\mathrm{OA} / \mathrm{core}}(\mathrm{mV})$ & $\zeta_{\mathrm{CS} / \mathrm{OA} / \mathrm{core}}(\mathrm{mV})$ \\
\hline $\mathrm{HD}$ & $1 / 1$ & $3 / 2$ & 3.19 & 0.17 & -20.8 & +25.7 \\
$\mathrm{LD}$ & $4 / 1$ & $3 / 2$ & 1.73 & 0.07 & -31.3 & +39.8 \\
\hline \hline
\end{tabular}


experiments, on dried powder samples and by calorimetric measurements on colloidal samples under RF fields.

Specific magnetization $(M)$ as a function of applied magnetic field $(H)$ at room temperature was obtained using vibrating sample magnetometer (VSM) LakeShore 7404 operated with maximum applied fields $\mu_{0} H_{\max }=1.5 \mathrm{~T}$ and using a superconducting quantum (SQUID) magnetometer with $\mu_{0} H_{\max }=2.5 \mathrm{~T}$. The later was also used to achieve hysteretic magnetization loops at 10, 47, 100, 170, 240, and $290 \mathrm{~K}$ and $M$ dependence with $T$ under zero field cool (ZFC) and field cool (FC) protocols with $H_{D C}=8 \mathrm{kA} / \mathrm{m}$.

Time-dependent calorimetric experiments for determining SAR were conducted by exposing $1 \mathrm{~mL}$ of the aqueous suspensions, hold in a clear glass Dewar, to $260 \mathrm{kHz}$ and field amplitude varying in the range from 16 to $52 \mathrm{kA} / \mathrm{m}$. Field generator consists of a resonant RLC circuit Hüttinger $(2.5 / 300)$ with a water refrigerated 6 turns coil of $2.5 \mathrm{~cm}$ diameter. The temperature was sensed during the experiment with an optical fiber sensor placed in the sample centre. The sensor was connected to a calibrated signal conditioner (Neoptix) of an accuracy of $\pm 0.1^{\circ} \mathrm{C}$. Colloid temperature was kept below $50{ }^{\circ} \mathrm{C}$ in order to minimize solvent evaporation and prevent its destabilization.

The SAR values were calculated from the initial slope $\partial T / \partial t$ of experimental heating curves as

$$
S A R=\frac{C}{[x]} \frac{\partial T}{\partial t},
$$

where $C$ is the volumetric heat capacity of the solvent $\left(4.18 \mathrm{~J} / \mathrm{cm}^{3} \mathrm{~K}\right)$.

\section{RESULTS AND DISCUSSIONS}

XRD patterns of both colloids (not shown) display welldefined reflection lines belonging to cubic spinel structure (JCPDS \#19-629) indicating that MNPs are single phase and that neither CS nor OA incorporations alter the crystalline magnetic core.

Figure 1 displays FTIR-DRIFTS spectra of HD and LD colloids containing different $\mathrm{OA}$ and CS proportions and pure polymer. Characteristic peaks of CS could be distinguished in the spectra, such as those located at around $3440 \mathrm{~cm}^{-1}$, ascribed to $-\mathrm{OH}$ group, at $1620 \mathrm{~cm}^{-1}$ assigned to $\mathrm{N}-\mathrm{H}$ bending vibration and the peak of $1392 \mathrm{~cm}^{-1}$ associated to $-\mathrm{C}-\mathrm{O}$ stretching of primary alcoholic group in $\mathrm{CS} .^{29}$ Magnetite contribution, observed in both spectra, is evidenced by a band located at almost $570 \mathrm{~cm}^{-1}$ assigned to $\mathrm{Fe}-\mathrm{O}$ bonds. These results confirm integration of polymeric and magnetic components in the formation of stable magnetic colloids.

TEM micrographies of LD and HD nanoparticles are shown in Figure 2, spheroid-like forms are distinguished and atomic planes are resolved indicating good crystallinity. Figures 2(a) and 2(b) display imaging of HD sample, where MNPs seems to be composed of several magnetite cores recovered by a polymeric layer. A large polymeric particle containing several magnetite cores is clearly seen in Figure 2(a) and the polymeric coating can also be observed. As a

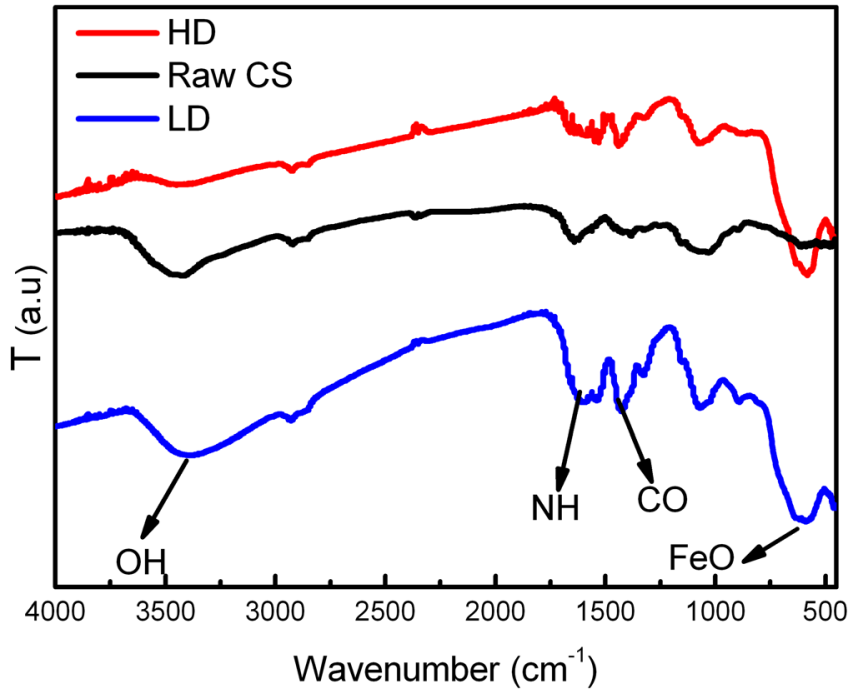

FIG. 1. FTIR-DRIFTS spectra of HD and LD magnetic colloids and raw chitosan. Transmittance $(T)$ as a function of the wavenumber $\left(\mathrm{cm}^{-1}\right)$. The spectra were shifted for clarity.

difference, LD sample imaging in Figures 2(d) and 2(e) shows almost individual MNPs where the CS layer seems to be acting as spacer between nanoparticles. From the images size histograms were constructed by counting MNPs from several micrographies of each sample. Mean size and s.d. of $9.7 \mathrm{~nm}\left(\sigma_{d}=4.5 \mathrm{~nm}\right)$ and $4.8 \mathrm{~nm}\left(\sigma_{d}=2.1 \mathrm{~nm}\right)$ were obtained for $\mathrm{HD}$ and LD samples by fitting the histograms with Gaussian functions (see Figures 2(c) and 2(f)). LD sample present lower sizes and narrower size distribution than HD sample, i.e., HD sample is more polydisperse than LD, consistently with the larger amount of OA used to synthesized LD. OA is used to limit growing after nucleation during synthesis protocol. On a qualitative analysis we observed that, although both samples were synthesized by the same route and dried for TEM measurements under the same conditions, HD sample presents some aggregates which may be due to interparticle interactions acting during dry treatment or to a permanent morphology of polymeric particles containing several particles as can be clearly seen in Figure 2(a). As already mentioned, HD sample displays on mean value larger sizes than LD, i.e., larger magnetic moment, resulting in a stronger dipolar interaction between nanoparticles favoring particles aggregation.

Surface properties were estimated by $\zeta$ measurements recorded on colloid precursors (OA-core nanoparticles) and are listed in the Table I for LD and HD suspension. Dispersions of OA-core in bidistilled water ( $\mathrm{pH}$ around 5.5) render negative $\zeta$ with magnitudes between $-20 \mathrm{mV}$ and $-31 \mathrm{mV}$. These values correlate with $\mathrm{OA}$ double layer and are in agreement with previous work. ${ }^{27} \mathrm{CS}$ incorporation causes positively charge surfaces attributed to surface exposed protonated amine groups on CS moieties. Z potential is considered as a measure of colloid stability. The recorded $\zeta$ magnitude between $+25 \mathrm{mV}$ and $+39 \mathrm{mV}$ correlates with stable suspensions.

According to the mechanism proposed in an earlier work, ${ }^{28}$ complexes acetate-CS are formed from acetic acid dissolving CS during nanoprecipitation step, and coordinate 


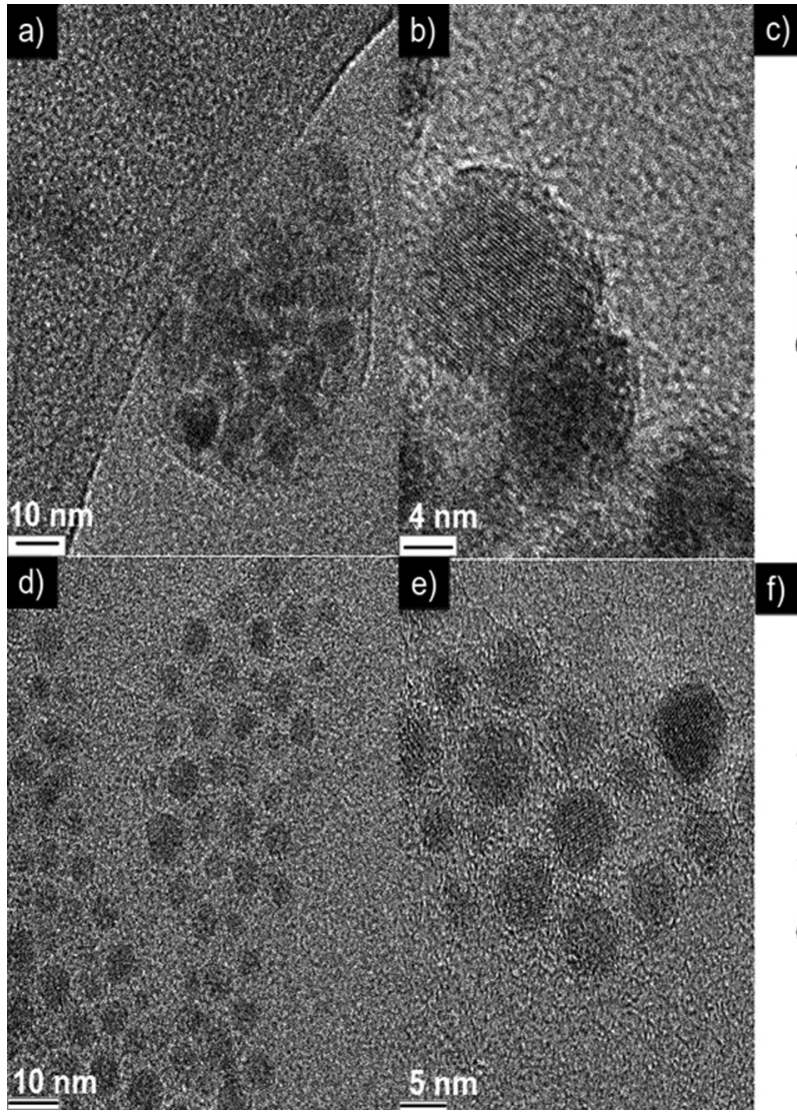

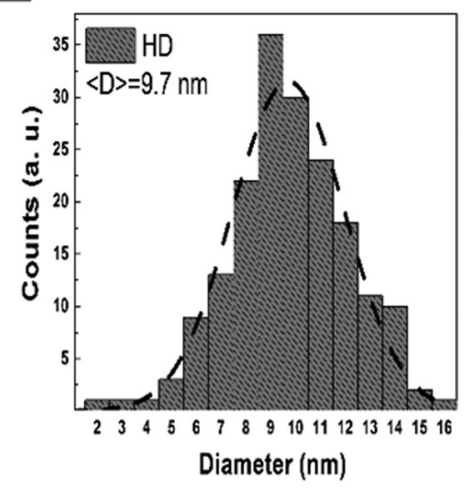

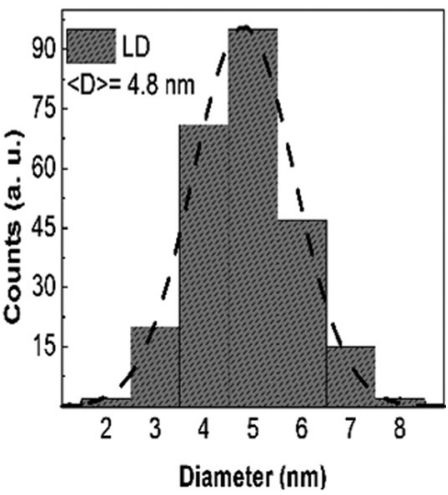

FIG. 2. Representative TEM images of HD sample are in (a) and (b); of LD sample in (d) and (f). Size histograms obtained by sampling 182 nanoparticles are shown in (c) for HD and in (f) for LD considering 250 nanoparticles. The dashed lines stand for a fitted Gaussian function. with surface $\mathrm{Fe}$ atoms of magnetite, leading to surface exposed $\mathrm{NH}_{3}{ }^{+}$groups. Therefore, colloid stabilization takes place by electrostatic repulsions.

A charged particle moving in a solvent drags a layer of fluid molecules and counter ions, resulting in an entity with a quite large hydrodynamic diameter $D_{H}$. In fact, measured $D_{H}$ values are larger than the overall nanoparticle size including coating. Mean values of number-average hydrodynamic sizes $\left(D_{H}\right)$ measured in bidistilled water are reported in Table II. LD colloid sample presents $\mathrm{D}_{\mathrm{H}}$ of $134.6 \mathrm{~nm}\left(\sigma_{D_{H}}=18.8 \mathrm{~nm}\right)$ while $\mathrm{HD}$ exhibits a lower value of $57 \mathrm{~nm}\left(\sigma_{D_{H}}=7.7 \mathrm{~nm}\right)$.

Scattering techniques like DLS and SAXS can be carried out in the suspension but only SAXS provides information on the internal structure of the scattering objects. Figure 3 shows the one-dimensional (1-D) SAXS pattern for the nanoparticles circularly averaged from the 2-D measured pattern. The 2-D pattern (not shown here) clearly revealed isotropic scattering, which is consistent with spherical objects scattering. The scattering curves shown in Figure 3 for $\mathrm{HD}$ and LD colloids are quite different. Two knees are observed for LD (see arrows in Fig. 3), indicating objects scattering at different length scales, while HD displays a more smooth decreasing $q$-behavior. HD pattern was well fitted considering spherical form factor weighted with a LogNormal size distribution (median $\log \left(d_{s}\right)$ and s.d. $\sigma_{s}$ ) convoluted with the analytic form of the structure function $S(q)^{30,31}$ derived for a fractal model of aggregation as described in Ref. 32. In this model, the power law form $\left(q^{-d_{f}}\right)$ of the scattering function is limited by finite cluster size $\xi$ and by the primary particle finite size $d_{s}$. SAXS analysis is indicating that the morphology seen by TEM for HD sample corresponds to a permanent aggregation with clusters of maximum size $\xi=28.2 \mathrm{~nm}$ inside larger CS particles of $48.6 \mathrm{~nm}$ size and not to a structuring produced during drying process. On the other hand, we have considered LD scattering curve as the superposition of the scattering from a dispersion of individual small magnetite nanoparticles which is observed at $q \geq 0.46 \mathrm{~nm}^{-1}$ and the scattering from a large CS particle which dominates scattering at $q$ values lower than $0.46 \mathrm{~nm}^{-1}$, being the magnetite nanoparticle and CS the two objects scattering at different length scale. The differential cross sections in the large $q$-range was fitted in the same way as HD sample but setting $S(q)=1$, which means no aggregation while in the low $q$-range with the unified equation of

TABLE II. Structural properties: $\langle d\rangle$ is the mean magnetite nanoparticle size deduced from TEM histograms and $\sigma_{d}$ the corresponding standard deviation (s.d); $D_{H}$ and $\sigma_{D_{H}}$ are the mean hydrodynamic size and s.d. from DLS data; SAXS: $d_{s}, \sigma_{\mathrm{s}}$ mean magnetite size and s.d, $d_{f}$ fractal exponent, $\xi$ cluster size and $d_{C S}$ chitosan particle size from SAXS data analysis.

\begin{tabular}{|c|c|c|c|c|c|c|c|c|c|}
\hline Label & $\langle d\rangle(\mathrm{nm})$ & $\sigma_{d}(\mathrm{~nm})$ & $D_{H}(\mathrm{~nm})$ & $\sigma_{D_{H}}(\mathrm{~nm})$ & $d_{s}(\mathrm{~nm})$ & $\sigma_{\mathrm{s}}(\mathrm{nm})$ & $d_{f}$ & $\xi(\mathrm{nm})$ & $d_{C S}(\mathrm{~nm})$ \\
\hline $\mathrm{HD}$ & 9.7 & 4.5 & 56.9 & 7.7 & 6.0 & 3.1 & 2.7 & 28.2 & 48.6 \\
\hline $\mathrm{LD}$ & 4.8 & 2.1 & 134.6 & 18.8 & 3.4 & 1.3 & - & - & 52.5 \\
\hline
\end{tabular}




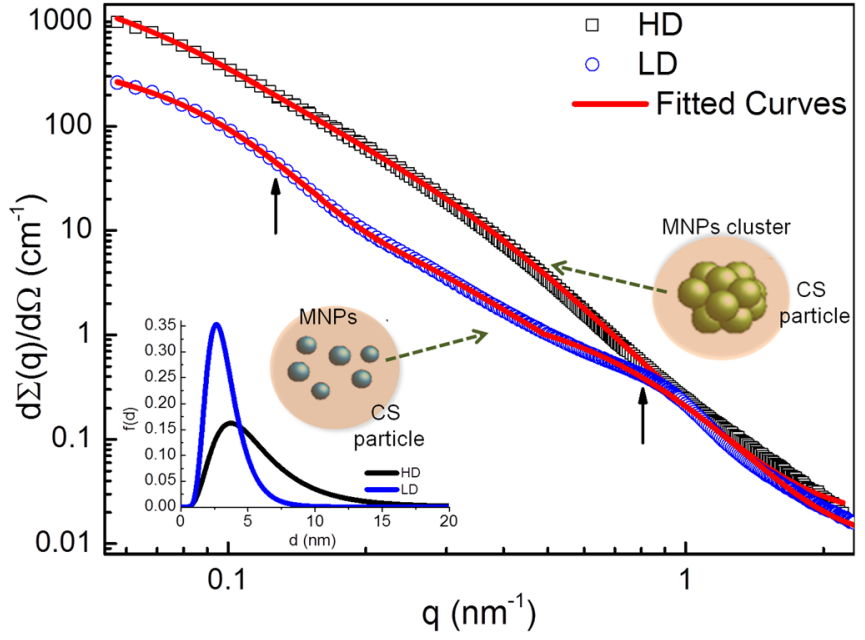

FIG. 3. 1D-SAXS patterns expressed as differential cross section $\mathrm{d} \Sigma(\mathrm{q}) / \mathrm{d} \Omega$ against scattering vector $\mathrm{q}$ for HD and LD colloids. Continuous lines stands for fitted curves using the models explained in the text. For each pattern, a schematic representation of MNPs structuring inside chitosan particles is plotted. Inset: $\mathrm{f}(\mathrm{d})$ is the lognorm magnetite core size distributions obtained from the fits.

Beaucage $^{33}$ for one structural level. This fit is consistent with a CS particle of $52.5 \mathrm{~nm}$. SAXS analysis in both cases is in agreement with TEM results and provides reliable values for CS coating and nanoparticles structuring. From this analysis, the overall size of the coated nanoparticles are $48.6 \mathrm{~nm}$ and $52.5 \mathrm{~nm}$ for $\mathrm{HD}$ and $\mathrm{LD}$ colloids. $\mathrm{D}_{\mathrm{H}}$ size, obtained in the suspension with DLS is calculated from the diffusion coefficient using the Stokes-Einstein and corresponds to the size of the dynamic hydrated particle. This value is close to the overall size for HD and 3 times larger for LD.

AFM images of LD and HD samples are shown in Figure 4, and some individual particles are labeled with their height (size). The topography clearly indicates spherical particles and sizes bellow $50 \mathrm{~nm}$ for both samples in agreement with SAXS results. The large shell of CS in LD nanoparticles is probably much less compact then drags a quite large layer of water and counterions.

This complete structural study involving four experimental techniques confidently indicates that in HD colloids each CS particle contain aggregates of several MNPs while in LD colloids the MNPs are spaced inside a large CS particle. A schematic draw is included in Figure 3.

Next, magnetic properties are evaluated using DC magnetometry techniques. The magnetization temperature dependence under ZFC $\left(M_{Z F C}\right)$ and FC $\left(M_{F C}\right)$ protocols are shown in Figure 5. These curves, displaying magnetization measurements recorded at a low DC field, while temperature is increase at a constant rate, are dominated by magnetic anisotropy and dipolar interactions rather than Zeeman interaction. $M_{Z F C}$ is consistent with a broad energy barrier distribution and peaks at $T_{m}$ close to $196 \mathrm{~K}$ and $71 \mathrm{~K}$ for $\mathrm{HD}$ and LD nanoparticles, respectively. For HD, the ZFC peak is wider and more shifted to higher temperatures consistently with larger size, broader distribution and stronger interactions between MNPs. For LD sample $M_{F C}$ increase with decreasing temperatures below the temperature of
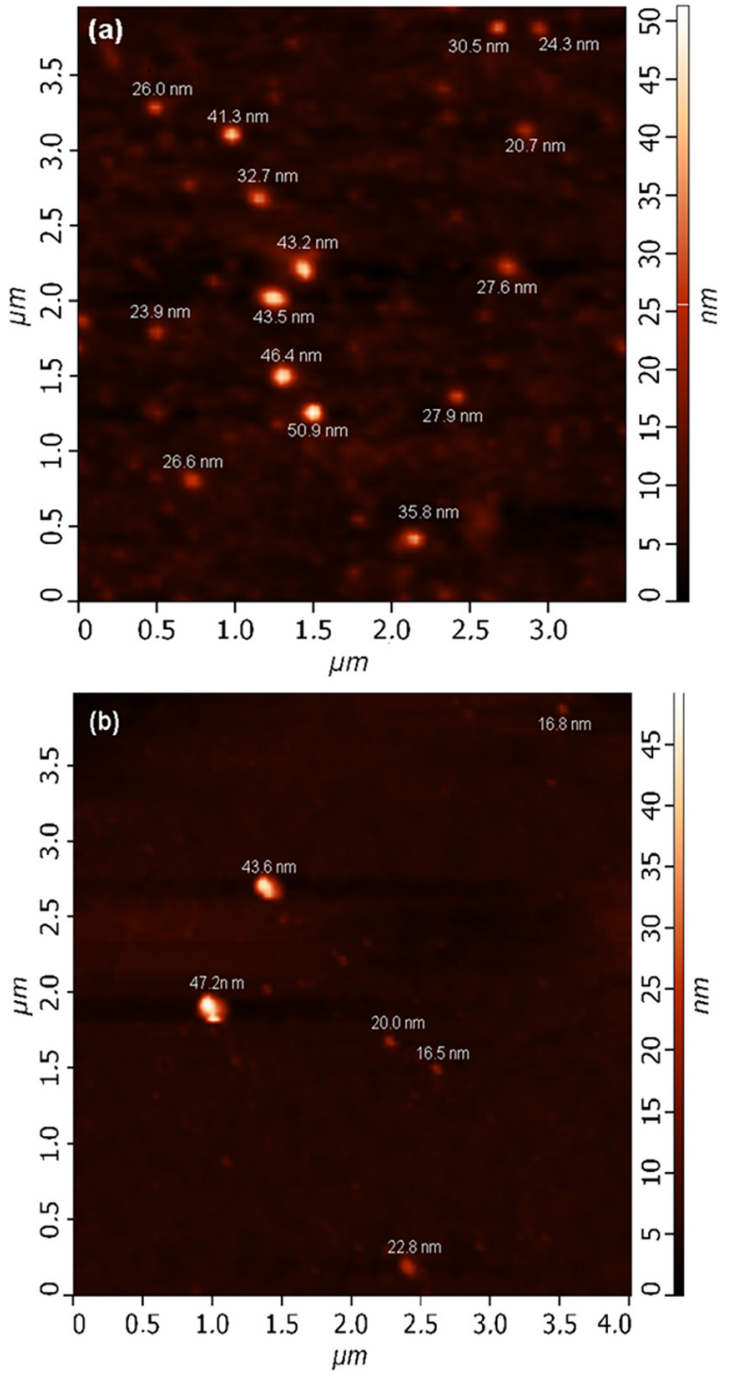

FIG. 4. AFM images (topography) of HD sample (a) and LD sample (b), some nanoparticles are labeled with their height (size).

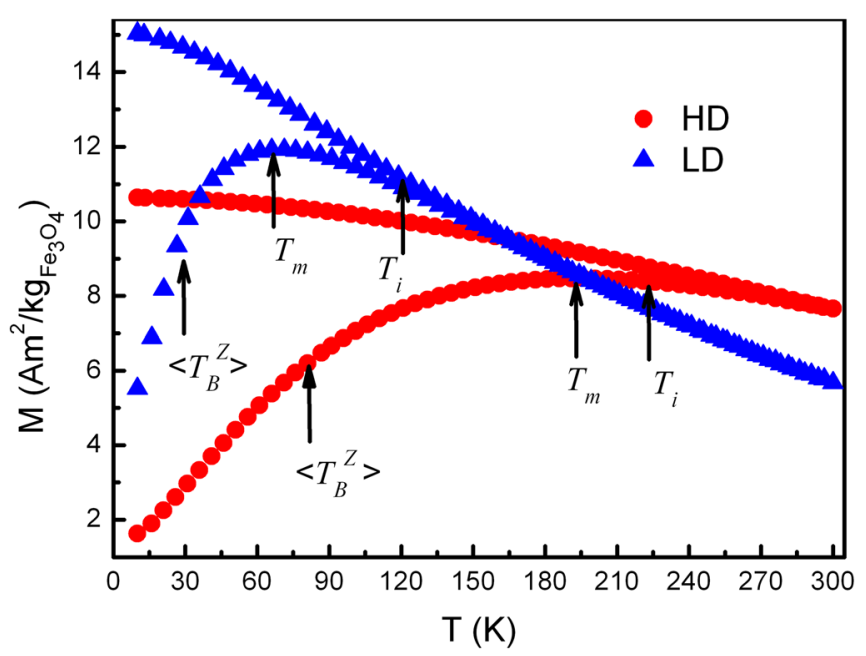

FIG. 5. Specific Magnetization $(M)$ against temperature $(T)$ measured under zero-field-cooling (ZFC) and field-cooling (FC) protocols with $H_{D C}=8 \mathrm{kA} / \mathrm{m}$ and heating rate of $5 \mathrm{k} / \mathrm{min} .\left\langle T_{B}^{Z}\right\rangle$ indicates mean blocking temperature obtained from $d\left(M_{F C}-M_{Z F C}\right) / d T, T_{i}$ the irreversibility temperature and $T_{m}$ the maximum of $M_{Z F C}(T)$ curve. 
TABLE III. Magnetic properties: $\langle\mu\rangle$ is the nanoparticle mean magnetic moment, $M_{s}$ specific saturation magnetization of magnetite nanoparticle at room temperature, $K_{e}$ effective anisotropy constant and $\left\langle T_{B}^{H}\right\rangle$ deduced from coercive field temperature behavior and $T_{m}$ and $\left\langle T_{B}^{Z}\right\rangle$ are the maximum and mean blocking temperatures deduced from ZFC/FC magnetization data. Specific absorption rate SAR measured at $260 \mathrm{kHz}$ and $52 \mathrm{kA} / \mathrm{m}$ and expressed per Fe $\mathrm{O}_{4}$ unit mass. SAR_s are the simulated SAR values.

\begin{tabular}{lcccccccc}
\hline \hline Label & $\langle\mu\rangle\left(\mu_{\mathrm{B}}\right)$ & $M_{S}\left(\mathrm{Am}^{2} / \mathrm{kg}\right)$ & $K_{e}\left(\mathrm{~kJ} / \mathrm{m}^{3}\right)$ & $T_{m}(\mathrm{~K})$ & $\left\langle T_{B}^{H}\right\rangle(\mathrm{K})$ & $\left\langle T_{B}^{Z}\right\rangle(\mathrm{K})$ & $\mathrm{SAR}(\mathrm{W} / \mathrm{g})$ & $\mathrm{SAR} \_\mathrm{S}(\mathrm{W} / \mathrm{g})$ \\
\hline $\mathrm{HD}$ & 8053 & 46 & $14.7_{4}$ & 196 & $83_{13}$ & $92.9_{1.7}$ & $82.7_{1.0}$ & 79 \\
$\mathrm{LD}$ & 4009 & 53 & $11.6_{9}$ & 71 & $31_{12}$ & $29.6_{0.2}$ & $8.2_{3.0}$ & 0.1 \\
\hline \hline
\end{tabular}

irreversibility $\left(T_{i}\right)$, while a flat behavior is observed for HD which is again an indication of HD as a more interacting system. In a previous work carried out on magnetite nanoparticles coated with citric acid a correlation between higher SAR values for larger $T_{m}$ values has been observed. ${ }^{13}$ Mean blocking temperature $\left\langle T_{B}^{Z}\right\rangle$ is obtained ${ }^{34}$ from the energy barrier distribution $f(T) \propto d\left(M_{F C}-M_{Z F C}\right) / d T$. Values are listed in Table III and indicated with arrows in Figure 5.

Hysteresis loops were recorded in both samples at various temperatures below $300 \mathrm{~K}$. Curve shape and behavior are similar for both samples then results are only shown for HD sample in Figure 6. For the data analysis, each sample is considered as an ensemble of MNPs with magnetocrystalline anisotropy axes randomly distributed. For magnetic data analysis, coating only contribute establishing how close the particles can be to each other, then the particles are considered as composed by a magnetic single domain core of volume $V$ and magnetic moment $\mu$ surrounded by a dead magnetic layer of thickness $\delta$. The loops were fitted using a displaced Langevin function weighted with a lognorm distribution of magnetic moments $g(\mu)$ plus a high field susceptibility $\chi_{a}$ linear term as it is described in a previous work. ${ }^{13}$ From this fitting procedure $M_{s}$, coercive field $H_{c}$, mean magnetic moment $\langle\mu\rangle$, s.d. of $\ln (\mu)$ variable $\sigma$, and $\chi_{a}$ are obtained. Relevant data is shown in Table III. Nanoparticle sizes determined from $g(\mu)$, using $\mu=M_{s} \rho V$ (mass density $\rho=5.18 \mathrm{~g} / \mathrm{cm}^{3}$ ), results in mean sizes smaller than TEM values and SAXS. We used mean values from histograms in Figures 2(c) and 2(f) to model SAR, because TEM is a direct

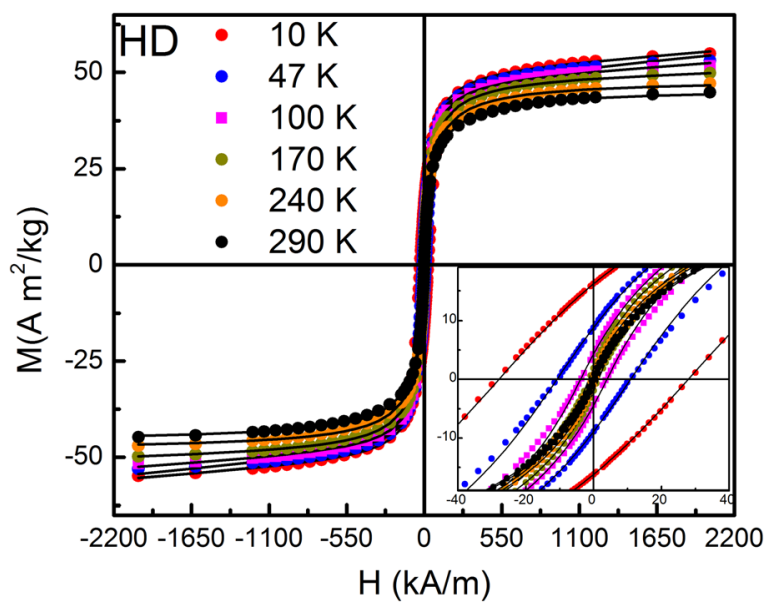

FIG. 6. Hysteresis loops of HD sample recorded at various temperatures. Inset shows a magnification to detail coercive field $\left(H_{c}\right)$ decrease with increasing measurement temperature. Solid lines are the best fits achieved using a displaced Langevin function weighted with a lognorm distribution of magnetic moments plus high field susceptibility. observation of the magnetite nanoparticle and we were able to count around 200 particles of each sample ensuring reliable statistics.

For both colloids $M_{s}$ at room temperature is $57 \%$ lower that bulk value ${ }^{6}(446 \mathrm{kA} / \mathrm{m})$ due to the presence of a dead magnetic layer $\delta \sim 1.8 \mathrm{~nm}$. This typical $M_{s}$ lowering is an undesired result because SAR depends on $M_{s}{ }^{2}$.

Mean magnetic moments $\langle\mu\rangle$ monotonically increases with $T$. This is an unphysical behavior commonly observed in dispersions behaving as interacting superparamagnets (ISP) as explained by Allia et al. ${ }^{35}$ In this regime, the particle moments interact through long-ranged dipolar random forces, whose effect is pictured in terms of a temperature $T^{*}$ added to measurement temperature in the denominator of the Langevin function argument. Consistently, magnetization data for samples perfectly scale with $H /\left(T+T^{*}\right)$ for $T>T^{*}$ and $\xi / \chi$ increase linearly with $T / M_{s}^{2}$ for both samples, being $\xi=\left\langle\mu^{2}\right\rangle /\langle\mu\rangle^{2}$ and $\chi$ the low field susceptibility. These plots confirming that the MNPs behave in the ISP regime are shown for HD sample in Figure 7.

The coercive field $\left(H_{c}\right)$ of an assembly of non interacting randomly oriented single-domain MNP exhibiting thermally activated coherent magnetization reversal follows the relation:

$$
H_{c}=0.48 \frac{2 K}{\mu_{0} M_{s}}\left(1-\left(\frac{T}{\left\langle T_{B}^{H}\right\rangle}\right)^{1 / 2}\right)
$$

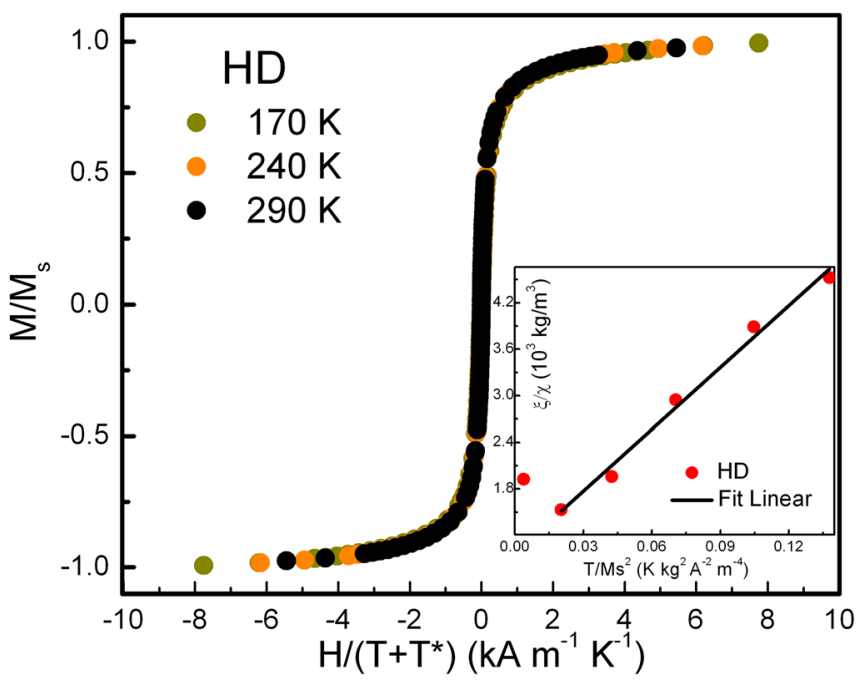

FIG. 7. (a) High temperature scaling regime for hysteresis loops displayed in Figure 6. Inset: plot displaying the linear behavior of $\xi=\left\langle\mu^{2}\right\rangle /\langle\mu\rangle^{2}$ (scale second moment of the magnetic moment) over $\chi$ (low field DC-susceptibility) against reduce variable $T / M s^{2}$, with $M s$ the saturation magnetization at the temperature $T$. 
below blocking temperature $\left\langle T_{B}^{H}\right\rangle,{ }^{36}$ where $K$ is the anisotropy constant. For interacting nanoparticles, the anisotropy constant is replaced by the effective anisotropy constant $K_{e}$. This constant takes into account the anisotropy energy of each particles and the interparticle interaction energy. $H_{c}$ values plotted against $T$ are shown in Figure 8 for LD and HD samples. $H_{c}$ monotonically decrease with $T$, but the decay is slower for HD sample. The curves were well fitted with $T^{1 / 2}$ dependence, and $\left[K_{e},\left\langle T_{B}^{H}\right\rangle\right]$ were used as fitting parameters, while $M_{s}$ was fixed at the values measured at $10 \mathrm{~K}$. The values $\left[14.7 \pm 0.4 \mathrm{~kJ} / \mathrm{m}^{3}, 83 \pm 13 \mathrm{~K}\right]$ and $[11.6$ $\left.\pm 0.9 \mathrm{~kJ} / \mathrm{m}^{3}, 31 \pm 12 \mathrm{~K}\right]$ were obtained for $\mathrm{HD}$ and $\mathrm{LD}$, respectively. The fitted $K_{\mathrm{e}}$ values are larger than magnetite bulk anisotropy constant ${ }^{37}$ of $9 \mathrm{~kJ} / \mathrm{m}^{3}$ at $300 \mathrm{~K} .\left\langle T_{B}^{H}\right\rangle$ values are in good agreement with those derived from $\mathrm{ZFC} / \mathrm{FC}$ data analysis. The energy barrier increases by increasing interparticle interactions strength and a shift of blocking temperature towards larger values is observed. Related models have been extensively discussed in Ref. 38. Using a rough estimation for the interparticle interaction strength as $E_{d}=\left(K_{e}-K\right) V$, HD is 18 times more interacting than LD. This result agrees, considering the dipolar character of the interparticle interaction proportional to the inverse cube of interparticle-distance, with the structural evidence of MNP clusters inside a given CS particle for HD sample where the MNPs are much closer than in the LD case where individuals particles spaced inside a CS particle as happens in LD sample.

Then, SAR values were determined for both colloids using Eq. (3). SAR of HD colloid is 10 times larger than for $\mathrm{LD}$ at $260 \mathrm{kHz}$ and $51.76 \mathrm{kA} / \mathrm{m}$. Recently, the largest SAR values, one order of magnitude higher than for isolated single-domain maghemite nanoparticles, have been reported for $50 \mathrm{~nm}$ particles composed by several smaller particles. ${ }^{39}$

The dependence of SAR with field amplitude is shown in Figure 9(a) and the typical heating curves for HD colloid are shown in Figure 9(b). The larger the field amplitude $H_{0}$ the faster the achieved heating, as expected from the quadratic dependence of SAR with $H_{0}$ (see Eq. (1)). The heating rate of LD colloid was measured at 49.01 and $51.76 \mathrm{kA} / \mathrm{m}$ and no significant temperature increase was observed by increasing the field amplitude.

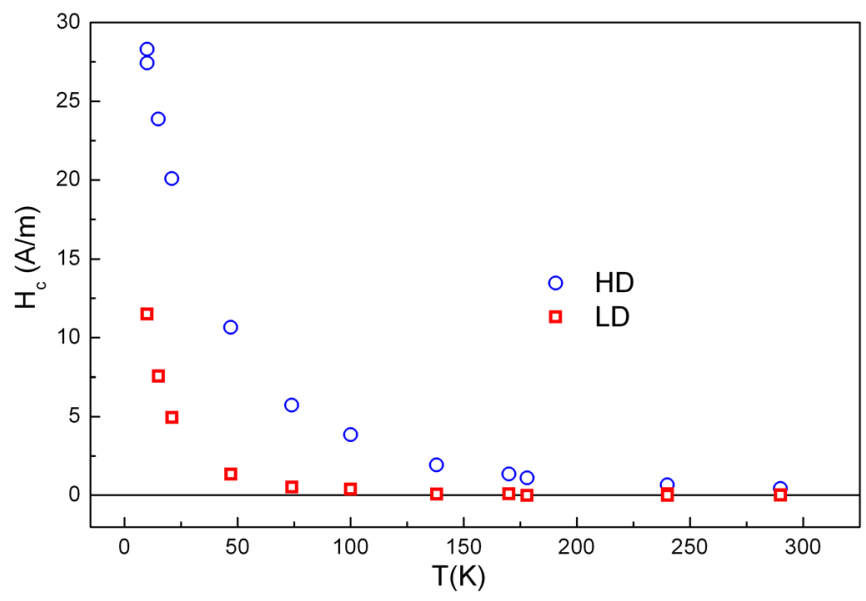

FIG. 8. Coercive field $\left(H_{c}\right)$ dependence on measurement temperature $T$.
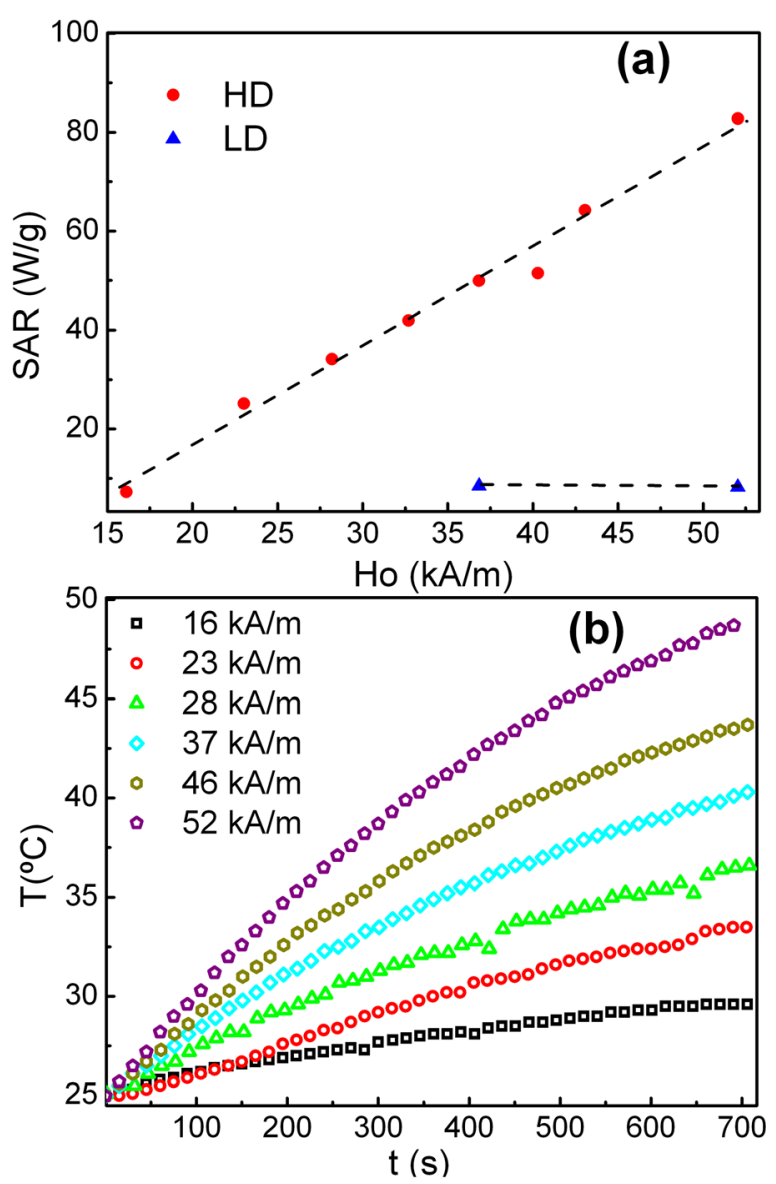

FIG. 9. (a) Specific absorption rate (SAR) derived from calorimetric measurements against excitation field amplitude $H_{0}$ obtained under RF frequency of $260 \mathrm{kHz}$. (b) Heating curves of HD sample.

As already mentioned Eq. (1) holds for single-size isolated particles, i.e., a non-interacting collection of monodispersed nanoparticles. Here, we are analyzing colloids displaying broad size distributions and behaving as interacting superparamagnets, meaning that relaxation times are distributed with a relaxation time distribution function $g(\tau)$ due to size distribution and the energy barrier may be changed by interparticles interaction. When the MNP assembly is monodispersed, the whole set of particle are fluctuating. Then, within a mean field theory, the field produce by dipolar interactions is a fluctuating field which average to zero. For broad size dispersion, the smaller particles are fluctuating, while the larger particles are blocked. The larger particles produce a static mean dipolar-field, increasing the energy-barrier height. In the present analysis, the dipolar interaction is included as a large effective anisotropy constant $K_{e}$ derived from DC magnetometry data, i.e., this $K_{e}$ value corresponds to MNPs inside the assembly. The obtained $K_{e}$ magnitudes are in concordance with the structuring of the MNPs inside the CS particle.

Then SAR is given by $S A R=\mu_{0} \pi f H_{o}^{2} \int \chi^{\prime \prime}(f, \tau) g(\tau) d \tau$ and making explicit $\tau$ size dependence is given by

$$
S A R=\mu_{0} \pi f H_{o}^{2} \frac{\int_{0}^{\infty} \chi^{\prime \prime}(D) V p_{N}(D) d D}{\int_{0}^{\infty} V p_{N}(D) d D},
$$


where $p_{N}(D) d D$ is the number of spherical particles with diameters between $D$ and $D+d D$. The main MNPs properties listed in Tables II and III were used to address the goodness of Eq. (5) to predict heating efficiency under RF fields of polydispersed MNPs of large hydrodynamic volume. Néel and Brown relaxation times, calculated using data in Tables II and III, are around $10^{-10} \mathrm{~s}$ and $10^{-4} \mathrm{~s}$, respectively. This large difference assures that the nanoparticles in both colloids magnetically relax by Néel mechanism and the possibility of a fraction of particles dissipating by Neél and the complement by Brown is excluded. Equation (5) was evaluated, for both colloids at $H_{0}=52 \mathrm{kA} / \mathrm{m}$ and $f=260 \mathrm{kHz}$, using the size distribution function $p_{N}(D)$ retrieved from TEM histograms jointly with $V_{H}$ from DLS and $K_{e}$ from DC magnetometry (i.e., isothermal magnetization), $\eta=10^{-3} \mathrm{~Pa}$.s and $\tau_{0}=10^{-10} \mathrm{~s}$. The attempt time value was choose from the detail analysis of Dormann et al. ${ }^{40}$ who showed that for interacting nanoparticles the dependence of $\log (\tau)$ against $1 / T_{B}$ departs from Arrhenius law and $\tau$ asymptotically goes to $\tau_{0}=10^{-10}$ s. By this way, Eq. (5) gives SAR values of 78.8 and $0.1 \mathrm{~W} / \mathrm{g}$ for $\mathrm{HD}$ and $\mathrm{LD}$, respectively. The agreement with calorimetric SAR data (also listed in Table III) is very good for HD sample not for $\mathrm{LD}$. However, this calculation results in low values for the low dissipating sample and the correct high values for high dissipating sample, i.e., the right behaviour is achieved.

Our analysis suggest that Eq. (5) combined with a complete set of structural and magnetic parameter $\left[\langle d\rangle, \sigma_{d},\left\langle D_{H}\right\rangle\right.$, $\left.\sigma_{D H}, K_{e}, M_{\mathrm{s}}\right]$ reproduce the SAR behavior of colloids displaying large size polydispersity. This fact validates the use of Eq. (5) to predict the colloids performance under other RF field parameters and to draw tips to improve nanoparticles design for biomedical colloids. It is worth mentioning that our analysis differs from the one carried out in Ref. 41 where the relaxations times $\tau_{N}$ and $\tau_{B}$ were separately averaged using size and hydrodynamic size distribution, respectively, and replaced in Eq. (1), resulting in SAR calculated values one order of magnitude lower than the experimental ones.

Simulations of $\tau, \tau_{B}$ and $\tau_{N}$ against $D$ were obtained using parameters listed in Tables II and III and are shown for HD colloid in Figure 10(a). The crossover from Neél to Brown relaxation mechanism appears at $D=20 \mathrm{~nm}$ for HD and $D=22 \mathrm{~nm}$ for LD. Then, the whole dispersion of MNP composing the colloids studied here dissipates through Neél mechanism consistently with the large hydrodynamic volumes. In Figure 10(b), simulations of the out of phase susceptibility dependence on MNPs size $\chi^{\prime \prime}(\tau(D))$ at fixed frequency of $260 \mathrm{kHz}$ and amplitude of $52 \mathrm{kA} / \mathrm{m}$ and $p_{N}(D)$ are plotted together for both samples. It is clearly seen that only when $p_{N}(D)$ superimposed to a non null value of $\chi^{\prime \prime}(\tau(D))$ the sample dissipates. $\chi^{\prime \prime}(\tau(D))$ determines the fraction of dissipating MNP at a given frequency. For LD sample $\chi^{\prime \prime}(\tau(D))$ is not overlapping with $p_{N}(D)$, then almost no heating is expected. For HD colloid, there is some overlapping, only a fraction of particles are dissipating and SAR is 10 times larger. The particles of sizes larger than $6 \mathrm{~nm}$ represent $34 \%$ of the magnetite mass in HD colloid and generate $98 \%$ of dissipated energy. One way to improved SAR of HD

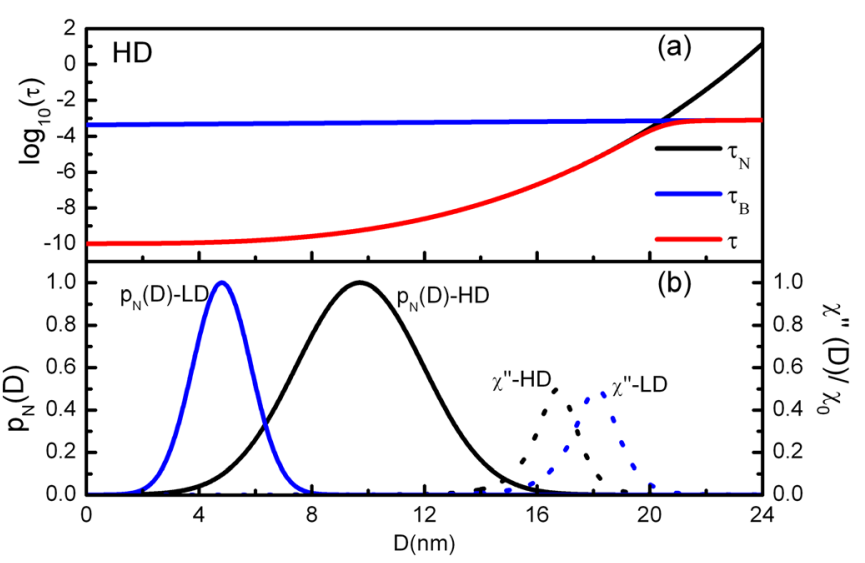

FIG. 10. (a) displays size dependence of $\tau, \tau_{N}$ and $\tau_{B}$ simulated for HD colloid using magnetic nanoparticle size from TEM, hydrodynamic volumes from DLS, effective anisotropy constant $K_{e}$ form DC-magnetometry, $\eta=10^{-3} \mathrm{~Pa}$.s and $\tau_{0}=10^{-10} \mathrm{~s}$. (b) displays Gaussian size distribution $\left(p_{N}(D)\right)$ against radii $(D)$ as derived from TEM data (filled lines) and simulation of the out of phase susceptibility $\chi^{\prime \prime}(D)$ normalized by DC-susceptibility $\chi_{0}$. The simulations were performed using $\tau(D)$ values plotted in (a) and other data listed in Tables II and III.

colloid would be to separate the nanoparticles on the basis of their dissipative properties. For instance, selecting the particles with radii larger than $6 \mathrm{~nm}$, SAR would be $225 \mathrm{~W} / \mathrm{g}$. Other way would be raising the field frequency because the maximum of $\chi^{\prime \prime}$ will shift to lower sizes. For instances, Eq. (5) evaluated at $f=500 \mathrm{kHz}$ predict an increase to $224 \mathrm{~W} / \mathrm{g}$ for HD colloid. Then, this analysis indicates that for colloids of MNPs with wide size distribution the largest dissipation would be achieved for an assembly of nanoparticles of mean size coincident with the maximum of $\chi^{\prime \prime}(D)$. Provided this coincidence condition the narrow the size distribution the largest the SAR in agreement with previous work. ${ }^{14,21,23}$ Other way, would be increasing the energy barrier height, i.e., increasing the phase shift between excitation wave and magnetization, which may be achieved in a controlled way by increasing interparticle interactions strength, for example, increasing the amount of particles inside the CS particle. But it should be kept in mind that increasing the energy barrier height the crossover in Fig. 10(a) will shift to lower sizes values and a situation corresponding to part of the dispersion (lower core sizes) dissipating by Néel and the rest (larger core sizes) by Brown mechanism could be reached. This analysis also indicates that for a MNP assembly displaying large polydispersity, the size optimization for improving heating efficiency must take into account both relaxation mechanisms and interparticles interactions.

Finally, we stress that an increase in size distribution width results in a decrease in SAR only in colloids in which mean MNP size $\langle d\rangle$ has been tailored to maximize the heat dissipation at the working conditions, i.e., tailored to fulfill the resonance condition $(2 \pi f \tau(\langle d\rangle)=1)$ at working frequency. When the mean size of the dispersion do not fulfill this condition, for instance, the mean size is located at the left of the maximum of $\chi^{\prime \prime}(D)$ as in Figure 10(b), increasing the polydispersity would result in SAR increase due to a large fraction of particles satisfying resonance condition. This reasoning can explain results of Ref. 9 were an increase 
in dipolar interaction strength was attempted by increasing size distribution without verifying that their mean sizes were satisfying resonance condition.

\section{SUMMARY AND CONCLUSIONS}

We have performed a complete structural and magnetic study of $\mathrm{Fe}_{3} \mathrm{O}_{4}$ magnetic nanoparticles coated with Oleic acid and chitosan to analyze their heating efficiency under radio frequency field for magnetic hyperthermia applications. We have studied two stable aqueous suspensions of magnetic nanoparticles displaying broad size distributions and differing markedly in the dispersed phase structure. One colloid consists of magnetic nanoparticles of mean size around $4.8 \mathrm{~nm}$ spaced inside chitosan particle of $52.5 \mathrm{~nm}$ and hydrodynamic size of $135 \mathrm{~nm}$. The other colloid consists of clusters of magnetic nanoparticles of mean size around $9.7 \mathrm{~nm}$ inside a chitosan particle of $48.6 \mathrm{~nm}$ and hydrodynamic sizes of $57 \mathrm{~nm}$. Our results indicate that both assemblies of suspended magnetic nanoparticles behave in the interacting superparamagnetic regime and the nanoparticles dissipate through Neél relaxation mechanism. It is shown that combining structural data with quasi-static magnetic measurement, the heating ability of a given colloid can be modeled within the framework of the linear response and Wohlfarth theories. This fact validate the use of the dynamic susceptibility modeled using structural and magnetic parameter derived from quasi-static measurements to predict heating ability of a given polydispersed particle suspension of large hydrodynamic sizes. We also conclude that dipolar interaction plays a key role being larger the dissipation for the more interacting nanoparticles assembly.

\section{ACKNOWLEDGMENTS}

This work has been funded by CONICET (PIP 01111), ANPCyT (PICT 00898), and UNLP-X11/556 grants of Argentina. Z-potential and XRD measurements were performed at INQUISUR, UNS-CONICET and CRIBABB (Bahia Blanca, Argentina); TEM at Brazilian Nanotechnology National Laboratory (LNNano), Centro Nacional de Pesquisa em Energia e Materiais (CNPEM), Brazil under the Project No. TEM-MSC-14825, SAXS at D01B-SAXS2 beam line of Brazilian Synchrotron Light Laboratory (LNLS) under proposal 13714. DC magnetometry using SQUID equipment of Red de Magnetismo y Materiales Magnéticos (RN3M). AFM at LFAyM of Instituto de Física La Plata (IFLP-CONICET). We thank F. H. Sánchez for helpful discussions on magnetism data analysis. Marcela B. Fernández van Raap and Pedro Mendoza Zélis are members of IFLP-CONICET, Veronica Lassalle is member of INQUISUR-CONICET, María Luján Ferreira is member of PLAPIQUI-CONICET, Diego Coral is fellow of COLCIENCIAS, Colombia and M. Elisa de Sousa and Paula Nicolás are fellows of CONICET, Argentina.

${ }^{1}$ N. R. K. Gilchrist, R. Medal, W. D. Shorey, R. C. Hanselman, J. C. Parrott, and C. B. Taylor, "Selective inductive heating of Limph," Ann. Surg. 146, 596 (1957).
${ }^{2}$ A. Jordan, P. Wust, R. Scholz, B. Tesche, H. Fähling, T. Mitrovics, T. Vogl, J. Cervós-Navarro, and R. Felix, "Cellular uptake of magnetic fluid particles and their effects on human adenocarcinoma cells exposed to AC magnetic fields in vitro," Int. J. Hyperthermia. 12(6), 705-722 (1996).

${ }^{3}$ R. Hergt, R. Hiergeist, M. Zeisberger, D. Schüler, U. Heyen, I. Hilger, and W. A. Kaiser, "Magnetic particle hyperthermia: nanoparticle magnetism and materials development for cancer therapy," J. Magn. Magn. Mater. 293, 80-86 (2005).

${ }^{4}$ M. V. Avdeev, B. Mucha, K. Lamszus, V. Ladislau, V. M. Garamus, A. V. Feoktystov, O. Marinica, R. Turcu, and R. Willumeit, "Structure and in vitro biological testing of water-based ferrofluids stabilized by monocarboxylic acids," Langmuir 26(11), 8503-8509 (2010).

${ }^{5}$ R. E. Rosensweig, Ferrohydrodynamics (Cambridge University Press, Cambridge, England, 1985).

${ }^{6}$ W. Brullot, N. K. Reddy, J. Wouters, V. K. Valev, B. Goderis, J. Vermant, and T. Verbiest, "Versatile ferrofluids based on polyethylene glycol coated iron oxide nanoparticles," J. Magn. Magn. Mater. 324, 1919-1925 (2012).

${ }^{7}$ T. T. Luong, T. P. Ha, L. D. Tran, M. Hung Do, T. Thu Mai, N. Hong Pham, H. Bich Thi Phan, G. Ha Thi Pham, N. My Thi Hoang, Q. T. Nguyen, and P. X. Nguyen, "Design of carboxylated Fe3O4/poly(styreneco-acrylic acid) ferrofluids with highly efficient magnetic heating effect," Colloids Surf. A: Physicochem. Eng. Aspects 384, 23-30 (2011).

${ }^{8}$ S. Laurent, S. Dutz, U. O. Häfeli, and M. Mahmoudi, "Magnetic fluid hyperthermia: focus on superparamagnetic iron oxide nanoparticles," Adv. Colloid Interface Sci. 166, 8-23 (2011).

${ }^{9}$ M. Jeun, S. Bae, A. Tomitaka, Y. Takemura, K. Ho Park, S. Ha Paek, and $\mathrm{K}$. W. Chung, "Effects of particle dipole interaction on the ac magnetically induced heating characteristics of ferrite nanoparticles for hyperthermia," Appl. Phys. Lett. 95, 082501 (2009).

${ }^{10}$ A. Urtizberea, E. Natividad, A. Arizaga, M. Castro, and A. Mediano, "Specific absorption rates and magnetic properties of ferrofluids with interaction effects at low concentrations," J. Phys. Chem. C 114(11), 4916-4922 (2010).

${ }^{11}$ D. Serantes, D. Baldomir, C. Martinez-Boubeta, K. Simeonidis, M. Angelakeris, E. Natividad, M. Castro, A. Mediano, D.-X. Chen, A. Sanchez, L. I. Balcells, and B. Martínez, "Influence of dipolar interactions on hyperthermia properties of ferromagnetic particles," J. Appl. Phys. 108, 073918 (2010).

${ }^{12}$ B. Mehdaoui, R. P. Tan, A. Meffre, J. Carrey, S. Lachaize, B. Chaudret, and M. Respaud, "Increase of magnetic hyperthermia efficiency due to dipolar interactions in low-anisotropy magnetic nanoparticles: Theoretical and experimental results," Phys. Rev. B 87, 174419 (2013).

${ }^{13}$ M. E. de Sousa María, M. B. Fernández van Raap, P. C. Rivas, P. Mendoza Zélis, P. Girardin, G. Pasquevich, J. Alessandrini, D. Muraca, and F. H. Sánchez, "Stability and relaxation mechanisms of citric acid coated magnetite nanoparticles for magnetic hyperthermia," J. Phys. Chem. C 117(10), 5436-5445 (2013).

${ }^{14}$ R. E. Rosensweig, "Heating magnetic fluid with alternating magnetic field," J. Magn. Magn. Mater. 252, 370-374 (2002).

${ }^{15}$ J. Carrey, B. Mehdaoui, and M. Respaud, "Simple models for dynamic hysteresis loop calculations of magnetic single-domain nanoparticles: Application to magnetic hyperthermia optimization,” J. Appl. Phys. 109, 083921 (2011).

${ }^{16}$ Rare Earth Magnetism: Structures and Excitations, edited by J. Jensen and A. R. Mackintosh (Clarendon Press, Oxford, 1991), Chap. 3.

${ }^{17}$ E. C. Stoner and E. P. Wohlfarth, "A Mechanism of magnetic hysteresis in heterogeneous alloys," Philos. Trans. Roy. Soc. A 240, 599-642 (1948).

${ }^{18}$ J. L. Dormann, L. Bessais, and D. Fiorani, "A dynamic study of small interacting particles: Superparamagnetic model and spin-glass laws," J. Phys. C: Solid State Phys. 21, 2015 (1988).

${ }^{19}$ E. Lima, Jr., T. E. Torres, L. M. Rossi, H. R. Rechenberg, T. S. Berquo, A. Ibarra, C. Marquina, M. R. Ibarra, and G. F. Goya, "Size dependence of the magnetic relaxation and specific power absorption in iron oxide nanoparticles," J. Nanopart Res. 15, 1654 (2013).

${ }^{20} \mathrm{P}$. Mendoza Zélis, G. A. Pasquevich, S. J. Stewart, M. B. Fernández van Raap, J. Aphesteguy, I. J. Bruvera, C. Laborde, B. Pianciola, S. Jacobo, and F. H. Sánchez, "Structural and magnetic study of zinc-doped magnetite nanoparticles and ferrofluids for hyperthermia applications," J. Phys. D: Appl. Phys. 46, 125006 (2013).

${ }^{21}$ J. P. Fortin, F. Gazeau, and C. Wilhelm, "Intracellular heating of living cells through Neél relaxation of magnetic nanoparticles," Eur. Biophys. J. 37, 223-228 (2008). 
${ }^{22}$ R. Hergt, S. Dutz, and M. Roder, "Effects of size distribution on hysteresis losses of magnetic nanoparticles for hyperthermia," J. Phys.: Condens. Matter 20, 385214 (2008)

${ }^{23}$ G. Salas, C. Casado, F. J. Teran, R. Miranda, C. J. Serna, and M. Puerto Morales, "Controlled synthesis of uniform magnetite nanocrystals with high-quality properties for biomedical applications," J. Mater. Chem. 22, 21065 (2012)

${ }^{24}$ J. Qu, G. Liu, Y. Wang, and R. Hong, "Preparation of $\mathrm{Fe}_{3} \mathrm{O}_{4}$-chitosan nanoparticles used for hyperthermia," Adv. Powder Technol. 21, 461-467 (2010).

${ }^{25}$ V. Belessi, R. Zboril, J. Tucek, M. Mashlan, V. Tzitzios, and D. Petridis, "Ferrofluids from magnetic-Chitosan hybrids," Chem. Mater. 20, 3298-3305 (2008).

${ }^{26}$ B. Gaihre, M. Seob Khil, D. Rae Lee, and H. Yong Kim, "Gelatin-coated magnetic iron oxide nanoparticles as carrier system: Drug loading and in vitro drug release study," Int. J. Pharam. 365, 180-189 (2009).

${ }^{27}$ V. Lassalle and M. Ferreira, "Nano and microspheres based on Polylactide (PLA) polymers and copolymers: An overview of their characteristics as a function of the obtention method," Macromol. Biosci. 7, 767-783 (2007).

${ }^{28}$ P. Nicolás, M. Saleta, H. Troiani, R. Zysler, V. Lassalle, and M. L. Ferreira, "Preparation of iron oxides nanoparticles stabilized with biomolecules: Experimental and mechanism issues," Acta Biomater. 9(1) 4754-4762 (2013).

${ }^{29}$ P. Li, A. M. Zhu, Q. L. Liu, and Q. G. Zhang, "Fe3O4/poly(N-isopropylacrylamide)/ chitosan composite microspheres with multiresponsive properties," Ind. Eng. Chem. Res. 47, 7700-7706 (2008).

${ }^{30}$ T. Freltoft, J. K. Kjems, and S. K. Sinha, "Power-law correlations and finite-size effects in silica particle aggregates studied by small-angle neutron scattering," Phys. Rev. B 33, 269-275 (1986).

${ }^{31} \mathrm{~S}$. Chen and J. Texeira, "Structure and fractal dimension of proteindetergent complexes," Phys. Rev. Lett. 57, 2583-2586 (1986).

${ }^{32}$ M. B. Fernández van Raap, P. Mendoza Zélis, D. F. Coral, T. E. Torres, C. Marquina, G. F. Goya, and F. H. Sánchez, "Self organization in oleic acid coated $\mathrm{CoFe}_{2} \mathrm{O}_{4}$ colloids: A SAXS study," J. Nanoparticle Res. 14(9), 1072-1075 (2012)

${ }^{33} \mathrm{G}$. Beaucage, "Approximations leading to a unified exponential/ power-law approach to small-angle scattering," J. Appl. Cryst. 28, 717-728 (1995).

${ }^{34}$ J. S. Micha, B. Dieny, J. R. Régnard, J. F. Jacquot, and J. Sort, "Estimation of the Co nanoparticles size by magnetic measurements in $\mathrm{Co} / \mathrm{SiO} 2$ discontinuous multilayers," J. Magn. Magn. Mater. 272-276, E967-E968 (2004).

${ }^{35}$ P. Allia, M. Coisson, P. Tiberto, F. Vinai, M. Knobel, M. A. Novak, and W. C. Nunes, "Granular $\mathrm{Cu}-\mathrm{Co}$ alloys as interacting superparamagnets," Phys. Rev. B 64(14), 144420 (2001).

${ }^{36}$ W. C. Nunes, W. S. D. Folly, J. P. Sinnecker, and M. A. Novak, "Temperature dependence of the coercive field in single-domain particle systems," Phys. Rev. B 70, 014419 (2004).

${ }^{37}$ K. Gilmore, Y. U. Idzerda, M. T. Klem, M. Allen, T. Douglas, and M. Young, "Surface contribution to the anisotropy energy of spherical magnetite particles," J. Appl. Phys. 97, 10B301 (2005).

${ }^{38}$ J. L. Dormann, D. Fiorani, and E. Tronc, "On the models for interparticle interactions in nanoparticle assemblies: comparison with experimental results," J. Magn. Magn. Mater. 202, 251-267 (1999).

${ }^{39}$ P. Hugounenq, M. Levy, D. Alloyeau, L. Lartigue, E. Dubois, V. Cabuil, C. Ricolleau, S. Roux, C. Wilhelm, F. Gazeau, and R. Bazzi, "Iron oxide monocrystalline nanoflowers for highly efficient magnetic hyperthermia," J. Phys. Chem. C 116(29), 15702-15712 (2012).

${ }^{40}$ J. L. Dormann, D. Fiorani, and E. Tronc, "Magnetic relaxation in fineparticle systems," in Advances in Chemical Physics, edited by I. Prigogine and S. A. Rice (John Wiley \& Sons, Inc., Hoboken, NJ, USA, 2007), Vol. 98, Chap. 4, p. 339.

${ }^{41}$ G. Vallejo-Fernandez, O. Whear, A. G. Roca, S. Hussain, J. Timmis, V. Patel, and K. O'Grady, "Mechanisms of hyperthermia in magnetic nanoparticles,” J. Phys. D: Appl. Phys. 46, 312001 (2013). 